\title{
Genetic susceptibility to aminoglycoside ototoxicity: How many are at risk?
}

Hsiao-Yuan Tang, $P h D^{1}$, Eldridge Hutcheson, $P h D^{3}$, Susan Neill, $P h D^{4}$, Margaret Drummond-Borg, MBChB, $M D^{5}$, Michael Speer, $M D^{2}$, and Raye Lynn Alford, $P h D^{1}$

Purpose: To assess the occurrence of two mutations associated with susceptibility to aminoglycoside ototoxicity.
Methods: Genetic analysis of anonymized, residual diagnostic specimens. Results: One occurrence of the A1555G
mutation and seven occurrences of the $961 \mathrm{delT}+\mathrm{C}(\mathrm{n})$ nucleotide change were found. Two previously unreported
sequence changes, T961G and $956-960 \mathrm{insC}$, were also found in six and five specimens, respectively. Conclu-
sions: Genetic susceptibility to aminoglycoside ototoxicity may be more common than previously suspected.
Further study of the 961 delT $+\mathrm{C}(\mathrm{n})$ mutation is recommended to confirm its role in aminoglycoside ototoxicity and
assess penetrance and variability with and without exposure to aminoglycoside antibiotics. Genet Med 2002:4(5):

$336-345$.

Key Words: aminoglycoside ototoxicity, hearing loss, deafness, A1555G, ototoxicity

Aminoglycoside-induced ototoxicity is a potentially preventable form of acquired hearing loss. Two mutations in the mitochondrial $12 \mathrm{~S}$ rRNA gene have been previously reported to predispose carriers to aminoglycoside-induced ototoxicity. ${ }^{1-7}$ One of these mutations, A1555G, has also been reported in hearing-impaired patients with no history of exposure to aminoglycoside antibiotics, suggesting that in some carriers of this mutation aminoglycoside exposure is sufficient, but not necessary, for hearing loss to occur, and additional environmental and/or genetic risk factors likely exist. ${ }^{1,2,8-13}$

A second mutation, the deletion of the $\mathrm{T}$ nucleotide at position 961 of the mitochondrial 12S rRNA gene, combined with a heteroplasmic, variable increase in the number of $\mathrm{C}$ nucleotides surrounding position 961, has been reported in one family and in an isolated patient with hearing loss and a history of exposure to aminoglycoside antibiotics. ${ }^{3,4}$ This mutation will be referred to herein as 961delT $+\mathrm{C}(\mathrm{n})$.

The documentation of these two susceptibility mutations raises questions about whether and how to screen for susceptibility to aminoglycoside-induced hearing loss. One way to assess individual risk is by careful evaluation of family history. Fischel-Ghodsian et al. ${ }^{14}$ documented the importance of thoroughly assessing a patient's family history for hearing loss prior

\footnotetext{
From ${ }^{1}$ The Bobby R. Alford Department of Otorhinolaryngology and Communicative Sciences and ${ }^{2}$ Department of Pediatrics, Baylor College of Medicine, Houston Texas; and ${ }^{3}$ Biochemistry and Genetics Division, ${ }^{4}$ Bureau of Laboratories, and ${ }^{5}$ Genetics Screening and Case Management Division, Texas Department of Health, Austin, Texas.

Raye Lynn Alford, PhD, The Bobby R. Alford Department of Otorhinolaryngology and Communicative Sciences, Baylor College of Medicine, One Baylor Plaza, NA102, Houston, TX 77030.

Received: April 3, 2002.

Accepted: June 21, 2002
}

DOI: 10.1097/01.GIM.0000029035.91778.53 to the administration of aminoglycoside antibiotics. Although the study population was very small, that study suggests that with careful assessment of family history, many cases could perhaps be prevented. However, the study also demonstrates that family history alone is not a perfect preventive method. Three of the seven patients in the study who experienced hearing loss after aminoglycoside exposure had no known family history of hearing loss. ${ }^{14}$

DNA-based mutation detection technologies offer a far more precise method for identification of at-risk individuals, but the appropriateness of screening for these two mutations and the circumstances under which screening should be conducted are not yet clear. Data are lacking on the general population frequency of these mutations in the United States. Prior studies have been conducted outside the United States for the A1555G mutation. However, because test groups were selected on the basis of hearing status, that is, either hearing or hearing-impaired populations, bias was introduced into the results and the studies failed to give an indication of the overall population incidence of the mutation. A single study ${ }^{15}$ reported screening an apparently randomly selected population in New Zealand. This study found one A1555G carrier in a sample of 206 persons. No studies have been done to determine the general population frequency of the $961 \mathrm{del} T+\mathrm{C}(\mathrm{n})$ mutation.

The A1555G mutation has been detected on a variety of mitochondrial haplogroups, in a variety of distinct populations, and in significant proportions of hearing-impaired patients and families. ${ }^{1-3,5-14,16}$ Those data suggest that the mutation has occurred multiple times and in several lineages throughout human history, that it is not an uncommon cause of hearing loss, and that a variety of populations may be at risk. Combined, those data suggest that the seemingly rare detection of the A1555G mutation may represent more of an ascer- 
tainment bias than a scarcity of the mutation itself, and that the frequency of this mutation in US populations warrants further study. ${ }^{1,2,5-14,16}$ Similar data for the 961delT + C(n) mutation are lacking.

Genetic testing for susceptibility to aminoglycoside ototoxicity is complicated by the fact that the A1555G mutation carries a risk for hearing loss without exposure to aminoglycoside antibiotics, albeit with reduced penetrance. ${ }^{1,2,8-12}$ As a result, screening for this mutation, in some cases, amounts to presymptomatic genetic testing. Similar data about additional effects of the 961delT $+C(n)$ mutation have not yet been reported.

Given the reduced use of aminoglycoside antibiotics in US populations compared with other populations, an important question is whether widespread screening may be appropriate or whether screening only high-risk populations would be a better approach. At-risk populations might be defined as those individuals with increased risk of exposure to aminoglycoside antibiotics because of some other, unrelated condition such as cystic fibrosis, an immunological dysfunction, or individuals in whom the use of aminoglycoside antibiotics is under consideration for treatment of infectious disease.

The overall population frequency of these mutations bears on the question of whether and how to screen for susceptibility. The purpose of this study was to assess the frequency of the A1555G and 961delT $+C(n)$ mutations in a large, unselected US population and to provide an estimation of the number of individuals at risk.

\section{MATERIALS AND METHODS}

\section{DNA samples}

The Newborn Screening Laboratory of the Texas Department of Health in Austin provided 1,173 anonymized dried blood spot cards for analysis. Specimens for this study were separated from a pool of residual diagnostic specimens which had been received by the Newborn Screening Laboratory through the Texas State Newborn Screening Program and were slated for destruction. The specimens were originally collected from newborns immediately after birth. All identifying marks and labels were removed from the specimens at the time of selection for this study. Specimens were selected entirely at random, without regard to gender, ethnicity, or region of the state from which the specimens were obtained. No information about the hearing status of the subjects or whether they have been exposed to aminoglycoside antibiotics is available. The anonymized specimens were shipped directly to the research laboratory in two separate batches. The short elapsed time between specimen batches eliminates the possibility that siblings, other than multiple births, are included in the specimen population. Upon arrival in the research laboratory at Baylor College of Medicine, the dried blood spot cards were assigned arbitrary identification numbers for the purposes of this study.

\section{Institutional review board (IRB) approval and informed consent}

This study used anonymized residual diagnostic specimens, originally collected for the Newborn Screening Program of the Texas Department of Health. Informed consent was not sought. This study was reviewed by the IRBs of both the Texas Department of Health and Baylor College of Medicine. The study was deemed exempt from further review by the Texas Department of Health IRB and approved by the Baylor College of Medicine IRB.

\section{DNA isolation}

Dried blood spots were processed according to manufacturer's specifications using the InstaGene ${ }^{\mathrm{TM}}$ Dry Blood Kit from Bio-Rad Laboratories (Hercules, CA).

\section{Polymerase chain reaction (PCR) for mutation analyses}

Processed dried blood spots were placed in 96-well PCR trays, with one spot per well. PCR was conducted with $50 \mathrm{pmol}$ of each primer, $10 \mathrm{mM}$ Tris- $\mathrm{HCl} \mathrm{pH} \mathrm{9.0,50} \mathrm{mM} \mathrm{KCl,} 1.5 \mathrm{mM}$ $\mathrm{MgCl}_{2}, 250 \mu \mathrm{M}$ dNTPs, and 1.6 U Taq DNA polymerase (Amersham Pharmacia Biotech Inc., Piscataway, NJ), in a total volume of $40 \mu \mathrm{L}$. Amplification was conducted as follows: $95^{\circ} \mathrm{C}$ for 5 minutes, followed by 40 cycles of $95^{\circ} \mathrm{C}$ for 1 minute, $55^{\circ} \mathrm{C}$ for 1 minute, and $70^{\circ} \mathrm{C}$ for 2 minutes in a thermal cycler (PTC100, MJ Research Inc., Waltham, MA).

Primers used for amplifying the mitochondrial DNA are as follows: sense 5'-GTCAATAGAAGCCGGCGTAA-3' (bases 920-939) and antisense 5'-ACTCTGGTTCGTCCAAGTGC-3' (bases 1607-1588).

The mitochondrial DNA reference sequence used for primer design was obtained from the National Center for Biotechnology web site (http://www.ncbi.nlm.nih.gov/GenBank Accession No. NC_001807: Homo sapiens mitochondrion, complete genome). The Human Mitochondrial DNA Revised Cambridge Reference Sequence ${ }^{17}$ was used for mutation/polymorphism analysis. This sequence was obtained from the Mitomap web site (http://www.gen.emory.edu/MITOMAP/mitoseq.html).

\section{Control DNA}

Positive control samples carrying the A1555G and 961delT $+\mathrm{C}(\mathrm{n})$ mutations were not available at the beginning of this study. Positive controls were made in the laboratory by PCR using primers containing either the normal or mutant sequence. The PCR conditions were the same as for amplifying the processed blood spots, as described in the previous section "Polymerase chain reaction (PCR) for mutation analyses," except that the annealing step was conducted at $50^{\circ} \mathrm{C}$.

The primers used for synthesis of the controls were as follows. For the A1555 normal control, sense 5'-AGAGGAGACAAGTCG-3' (bases 1548-1562), antisense 5'-GCTAAGGTTGTCTGGTAGTA-3' (bases 1720-1701); and for the A1555G mutant control, sense 5'-AGAGGAGGCAAGTCG-3' (bases 1548-1562, with A1555G change), antisense 5'-GCTAAGGTTGTCTGGTAGTA-3' (bases 1720-1701). For the 961delT + C(n) normal control, sense 5'-TCACCCCCTCCCCAA-3' 
(bases 953-967), antisense 5'-GGATATGAAGCACCGCCA-3' (bases 1191-1174); and for the 961delT mutant control, sense 5'-TCACCCCCCCCCAAT-3' (bases 953-968, with deletion of base 961), antisense 5'-GGATATGAAGCACCGCCA-3' (bases 1191-1174).

The DNA sequence of the synthetic controls was confirmed by DNA sequencing as described in the section titled "DNA sequencing."

\section{Dot blots}

Fifteen microliters of the specimen PCR reactions and $10 \mu \mathrm{L}$ of the control DNA PCR reactions were spotted onto Hybond-N+ nylon membrane (Amersham Pharmacia Biotech Inc.) using the Bio-Dot Microfiltration Apparatus (Bio-Rad Laboratories). The spotted membrane was air-dried for 15 minutes, then placed on a Whatman filter paper saturated with $0.4 \mathrm{M} \mathrm{NaOH}$ for 10 minutes and subsequently neutralized in 2 $\times$ SSC, $0.4 \mathrm{M}$ Tris- $\mathrm{HCl} \mathrm{pH}$ 7.5. Membranes were stored in the neutralization buffer at $4^{\circ} \mathrm{C}$ until used. Duplicate dot blots were prepared for each 96-well PCR reaction tray.

\section{Allele-specific oligonucleotide (ASO) hybridization}

Probe oligonucleotides (Genset Corp., La Jolla, CA) with the normal or mutant nucleotide in the middle position were labeled with a thermostable alkaline phosphatase via a crosslinker using the AlkPhos Direct Oligolabelling kit (Amersham Pharmacia Biotech Inc.). Hybridization was performed at $45^{\circ} \mathrm{C}$ for the $\mathrm{A} 1555 \mathrm{G}$ assay and at $55^{\circ} \mathrm{C}$ for the $961 \mathrm{delT}+\mathrm{C}(\mathrm{n})$ assay. Hybridization was performed in the buffer provided with the kit for at least 16 hours in a hybridization oven (Amersham Pharmacia Biotech Inc.). After hybridization, blots were washed as recommended by the manufacturer. The CDPStar ${ }^{\mathrm{TM}}$ chemiluminescent method (Amersham Pharmacia Biotech Inc.) was used for detection.

The oligonucleotide probe sequences used are as follows. For detection of the A1555G mutation: normal probe, 5'AGAGGAGACAAGTCG-3' and mutant probe, 5'-AGAGGAGGCAAGTCG-3' (bases 1548-1562). For detection of the 961delT $+C(n)$ mutation, antisense probes with the following sequences were used: normal probe, 5'-CTTTATTGGGGAGGGGGTGA-3' and mutant probe, 5'-CTTTATTGGGGGGGGGTGA-3' (bases 972-953).

Duplicate dot blots for each 96-well PCR reaction tray were screened first for the A1555G mutation: one filter screened with a normal probe, the duplicate filter with the mutant probe. After completion of the A1555G studies, blots were stripped and reprobed for the 961delT $+C(n)$ mutation.

\section{DNA sequencing}

Mutant specimens identified by ASO or specimens whose ASO results were equivocal were evaluated by DNA sequencing using $\mathrm{ABI}$ prism BigDye ${ }^{\mathrm{TM}}$ II or BigDye ${ }^{\mathrm{TM}}$ III kits (Applied Biosystems, Foster City, CA). The DNA sequence of the synthetic normal and mutant controls was also confirmed by fluorescent dideoxy sequencing using the ABI prism BigDye ${ }^{\mathrm{TM}} \mathrm{II}$ kit (Applied Biosystems). DNA sequence was analyzed on an ABI Prism 310 Genetic Analyzer (Applied Biosystems).

Specimens selected for DNA sequencing were reprocessed and reamplified. Primers used for PCR amplification and cycle sequencing of mutant or equivocal specimens and controls were as follows. For detection of nucleotide 1555: forward primer 5'-TGTAGCCCATGAGGTGGCAA-3' (bases 1335-1354), reverse primer 5'-GCTAAGGTTGTCTGGTAGTA-3' (bases 17201701). For detection of nucleotide 961: forward primer 5'-AGCATCAAGCACGCACGAAT-3' (bases 754-773), reverse primer 5'-GGATATGAAGCACCGCCA-3' (bases 1191-1174).

Cycle sequencing was conducted for 25 cycles as follows: $96^{\circ} \mathrm{C}$ for 20 seconds; $50^{\circ} \mathrm{C}$ for 10 seconds; $60^{\circ} \mathrm{C}$ for 4 minutes. After completion of 25 cycles, reactions were held at $4^{\circ} \mathrm{C}$.

\section{Restriction analysis}

Because of difficulty with detection of the 961delT $+\mathrm{C}(\mathrm{n})$ mutation using ASO methods in this and a previous study, ${ }^{3}$ remaining specimens that had not been subjected to DNA sequencing were evaluated for the presence of the 961delT + $\mathrm{C}(\mathrm{n})$ mutation by PCR and restriction digestion with the restriction enzyme MnlI (New England Biolabs, Beverly, MA). $\mathrm{MnlI}$ restriction analysis will detect both the 961delT $+\mathrm{C}(\mathrm{n})$ mutation and the T961G nucleotide substitution, but, because of its recognition sequence, $\mathrm{MnlI}$ is not able to distinguish between the wild type and 956-960insC sequences.

Specimens were amplified as described in the section "Polymerase chain reaction (PCR) for mutation analyses," except that a total reaction volume of $30 \mu \mathrm{L}$ was used, the annealing temperature was adjusted to $50^{\circ} \mathrm{C}$, and the following oligonucleotide primers were used: sense, 5'-AGCATCAAGCACGCAGCAAT-3' (bases 754-773) and antisense, 5'-GGATATGAAGCACCGCCA-3' (bases 1191-1174). This PCR reaction yields a product 438 base pairs in size. Seventeen and one-half microliters of each PCR reaction were incubated with 2 units of MnlI using manufacturer-supplied digestion buffer and according to the manufacturer's recommendations.

Digestion with $M n l I$ was expected, in specimens carrying either the normal sequence or the 956-960insC alteration, to yield fragments of 216, 146, and 76 base pairs in size. Specimens carrying either the $961 \mathrm{delT}+\mathrm{C}(\mathrm{n})$ or the T961G sequence changes, that interrupt the $5^{\prime}$-CCTC $\left(\mathrm{N}_{7}\right)-3^{\prime} / 3^{\prime}-$ GGAG(N)-5' consensus sequence for $M n l I$, are expected to remain uncut at position 969, resulting in fragments 362 and 76 base pairs in size. Digested samples were analyzed on $1.8 \%$ agarose gels.

\section{Haplogroup analysis of specimens carrying nucleotide substitutions at or around position 961}

Specimens found to carry the 961delT + C(n), T961G, or 956-960insC sequence changes were assessed for mitochondrial haplogroup using previously published methodologies and criteria. ${ }^{17-24}$ Dried blood spot specimens were reprocessed and amplified with previously published primer pairs, ${ }^{18,19}$ as needed for haplogroup analyses, except that the primer covering positions 5317-5333 was substituted with a primer cover- 
ing positions 6761-6782 to shorten the length of the PCR product and reduce the number of restriction sites contained within the PCR product. PCR products were digested with restriction endonucleases, as needed for haplogroup analyses, overnight, according to standard protocols and manufacturer's specifications, and using manufacturer-supplied digestion buffers. Restriction fragments were visualized by ethidium bromide agarose gel electrophoresis using standard methodologies.

Specimens were tested for and assigned to haplogroups ${ }^{17-24}$ as follows: Haplogroup A, gain of a HaeIII site at position 663; Haplogroup B, deletion of nine base pairs between positions 8269 (MTCO2) and 8295 (MTTK); Haplogroup F, loss of the HincII site at position 12406; Haplogroup $\mathrm{H}$, loss of the Alu I site at position 7025; Haplogroup I, loss of the HaeII site at position 4529; Haplogroups J and T, gain of an NlaIII site at position 4216; Haplogroups K and U, gain of a Hinfl site at position 12308; Haplogroup L, gain of a HpaI site at position 3592; Haplogroup M, gain of a DdeI site at position 10394 with gain of an AluI site at position 10397; Haplogroup T was confirmed by gain of a BamHI site at position 13366; Haplogroup V, loss of the NlaIII site at position 4577; Haplogroup W, gain of an AvaII site at position 8249 with loss of the HaeIII site at position 8994; and, Haplogroup X, loss of the DdeI site at position 1715 . Once assigned to a particular haplogroup, specimens were not further tested.

\section{RESULTS}

A total of 1,173 anonymized residual diagnostic specimens were obtained from the Newborn Screening Laboratory of the Texas Department of Health (Austin, Texas). Specimens that failed to PCR on the first attempt were repeated. Twelve specimens failed twice to amplify by PCR as indicated by lack of a spot on both the normal and mutant A1555/A1555G ASO filters. These specimens were counted as specimen failures and were not analyzed further.

As shown in Figure 1, A and B, one specimen was identified by ASO analysis to carry the A1555G mutation (Fig. 1B, sample well H7). This specimen was sequenced in both directions for confirmation. Figure 2A shows an example of the forward direction, wild type sequence for comparison. The DNA sequence of the specimen shown by ASO to carry the A1555G mutation, as shown in Figure 1B, is shown in Figure 2B (forward direction only, reverse direction sequence not shown). Ironically, this specimen was identified among the first 86 specimens tested. After confirmation, this specimen was used as a positive control on future ASO filters. Because of the clarity of the sequencing data (Fig. 2B), it is likely that this individual is homoplasmic, or nearly homoplasmic, for the mutation.

Figure 3, A and B, shows typical dot blots from the T961/ 961delT + C(n) ASO assay. For the T961/961delT + C(n) assay, the blots used in the A1555/A1555G assay were stripped and reprobed with the T961 wild type and 961delT $+\mathrm{C}(\mathrm{n})$ mutant probe oligonucleotides. No positive specimens were detected among the 1,161 specimens screened with the mutant
A

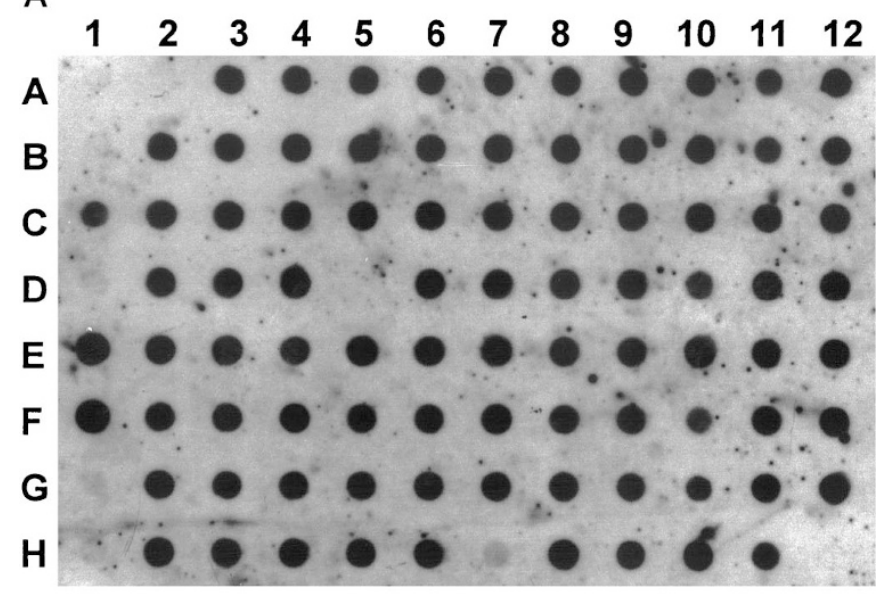

B

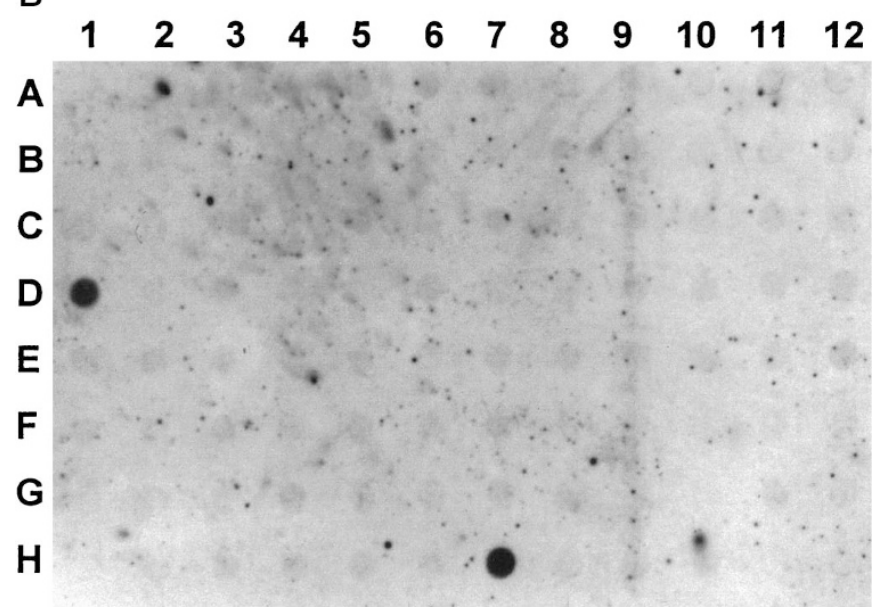

Fig. 1 ASO for the A1555G mutation. Duplicate blots screened with (A) the wild type or (B) the mutant probe. Controls are as follows: A1, 961 wild type; B1, 961 mutant; C1, A1555 wild type; D1, A1555G mutant; E1, F1, anonymous genomic DNA controls; G1, H1, water blanks; B2-G12, test specimens. A2, H12 are empty wells. Autoradiographs were scanned into the computer with a UMAX Astra 2400S Color Scanner (UMAX Technologies Inc., Fremont, CA). Scanned images were edited for brightness and contrast in Adobe Photoshop 6.0 (Adobe Systems Inc., San Jose, CA). The blots shown in this figure are not the same blots as those shown in Figure 3. The blots shown in this figure carry different specimens from the blots shown in Figure 3.

sequence $961 \mathrm{del} T+\mathrm{C}(\mathrm{n})$ probe. However, 25 poorly hybridizing specimens were noted on the blots screened with the T961 wild type sequence probe. Based on prior data from the A1555/A1555G screening process, these specimens were known to have amplified adequately by PCR and were expected to hybridize sufficiently. To clarify their failure to hybridize to the wild type probe as expected, these specimens were sequenced.

Of particular note on the blot shown in Figure 3A are the samples in wells C7, D7, E10, and G2. These specimens hybridized well to the wild type oligonucleotide on the A1555/ A1555G ASO test and were included in the 25 specimens selected for sequencing. The specimen in well $\mathrm{H} 7$ of Figure 3A, which appears to have hybridized poorly in the T961/961delT $+\mathrm{C}(\mathrm{n})$ assay, also hybridized poorly in the A1555/A1555G assay. This specimen was repeated on another blot. On repeat 
A

AC C CCT AC GC AT TTAT AT A G AG G AGACAAGTC GT AACAT GGTAA

B

AC C CCT ACGCATTTATATA G AGGAG GC AAGT CGT AACAT GGTAA

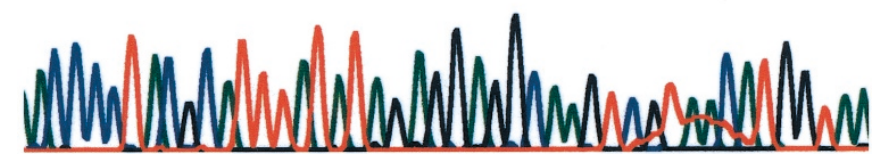

Fig. 2 Fluorescent dideoxy sequencing the ASO positive specimen shown in well $\mathrm{H} 7$ of Figure 1B. (A) Wild type specimen sequenced for comparison. (B) Specimen H7 from Figure 1B. Position 1555 is noted by the arrow in both A and B.

analysis, this specimen appeared to be of wild type sequence in both assays (data not shown). It is important to note that the blots in Figure 1 and the blots in Figure 3 are not the same blots. Therefore, H7 from Figure 1 is not H7 depicted in Figure 3.

Seven of the 25 specimens selected for sequencing after the T961/961delT $+C(n)$ ASO assay were found to contain the 961delT $+\mathrm{C}(\mathrm{n})$ mutation. DNA sequencing of a representative specimen is shown in Figure 4B. (Fig. 4A shows an example of the T961 wild type sequence for comparison.) Five specimens were found to carry a previously unreported ${ }^{17}$ sequence change: an extra $\mathrm{C}$ nucleotide, inserted in the run of $5 \mathrm{C}$ nucleotides immediately upstream of position 961 . This nucleotide change is referred to herein as 956-960insC (Fig. 4C). Six specimens were found to contain a distinct, but also previously unreported ${ }^{17}$ sequence change at position 961: a $\mathrm{T}$ to $\mathrm{G}$ transversion. This nucleotide change is referred to herein as T961G (Fig. 4D). One of these six specimens also carried a previously unreported ${ }^{17} \mathrm{G}$ to $\mathrm{C}$ transversion at position 951 (G951C, Fig. $4 \mathrm{E}$ ). The remaining 7 specimens of the 25 specimens selected for sequencing carried the wild type sequence at and around position 961 (data not shown).

Specimens C7 and D7 from the blot shown in Figure 3 were found to carry the 956-960insC sequence change. Specimen G2 from the blot shown in Figure 3 was found to carry the 961delT $+C(n)$ mutation. Specimen E10 from the blot shown in Figure 3 was found to carry the wild type sequence.

As a consequence of the questionable ability to detect the 961delT $+\mathrm{C}(\mathrm{n})$ mutation by ASO and a previously reported similar experience, ${ }^{3}$ the remaining 1,136 specimens were ex-
A

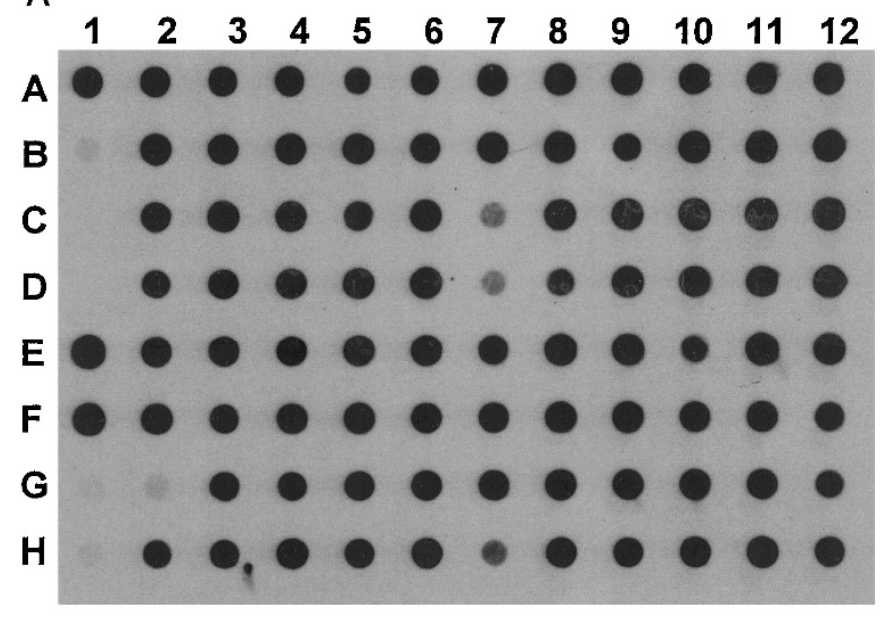

B

\section{$\begin{array}{llllllllllll}1 & 2 & 3 & 4 & 5 & 6 & 7 & 8 & 9 & 10 & 11 & 12\end{array}$}

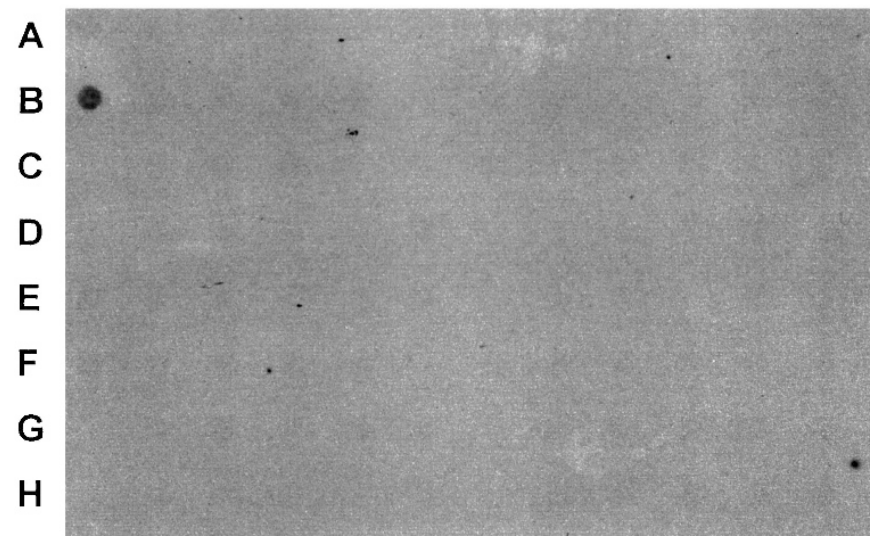

Fig. 3 ASO for the 961 delT $+C(n)$ mutation. Duplicate blots screened with (A) the wild type or (B) the mutant probe. Controls are as follows: A1, 961 wild type; B1, 961 mutant; C1, A1555 wild type; D1, A1555G mutant; E1, F1, anonymous genomic DNA controls; G1, H1, water blanks; A2-H12, test specimens. Autoradiographs were scanned into the computer with a UMAX Astra 2400S Color Scanner. Scanned images were edited for brightness and contrast in Adobe Photoshop 6.0. The blots shown in this figure are not the same blots as those shown in Figure 1. The blots shown in this figure carry different specimens from the blots shown in Figure 1.

amined by PCR and restriction digestion with the enzyme MnlI. This enzyme recognizes the consensus sequence 5'$\operatorname{CCTC}\left(\mathrm{N}_{7}\right)-3$ '. Specimens carrying the wild type sequence or the 956-960ins $\mathrm{C}$ sequence are digested by the enzyme, while specimens carrying the $\mathrm{T} 961 \mathrm{G}$ sequence or the $961 \mathrm{del} \mathrm{T}+\mathrm{C}(\mathrm{n})$ sequence remain uncut at position 969 (Fig. 5). All but eight specimens successfully reamplified for the MnlI assay. No additional occurrences of the 961delT $+\mathrm{C}(\mathrm{n})$ or T961G sequence alterations were found by $\mathrm{MnlI}$ restriction analysis. One specimen showed a restriction pattern consistent with the presence of a previously reported ${ }^{17} \mathrm{~T}$ to $\mathrm{C}$ polymorphism at position 1107. This polymorphism would be expected to generate bands of 216 and 222 base pairs in size upon MnlI digestion. The observed restriction pattern for this specimen suggests that there could be two bands of similar size that did not resolve during the short run time of the agarose gel (data not shown). 
A
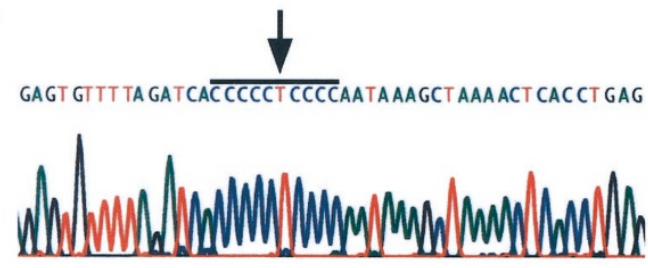

B

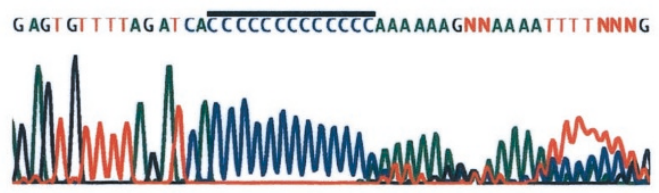

$\mathrm{C}$

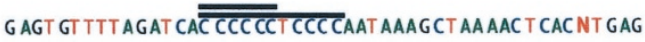

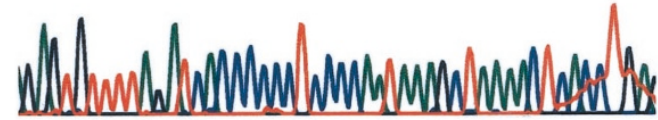

$\mathrm{D}$
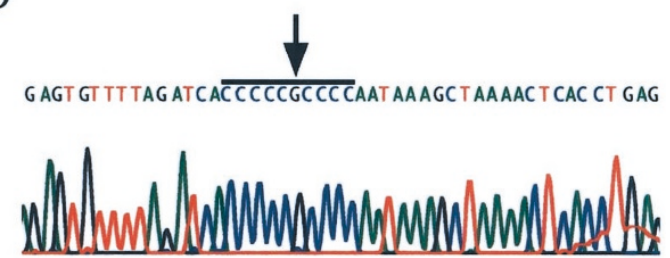

$\mathrm{E}$

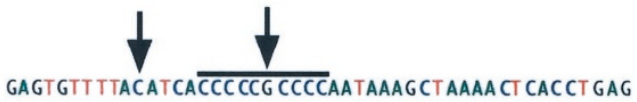

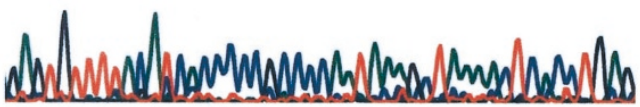

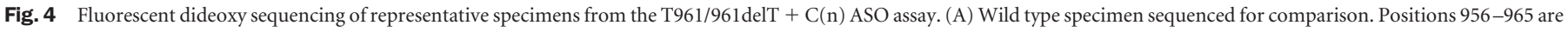
highlighted by a bar. Position 961 is noted by an arrow. (B) 961delT + C(n). (C) 956-960insC. (D) T961G. (E) T961G + G951C.

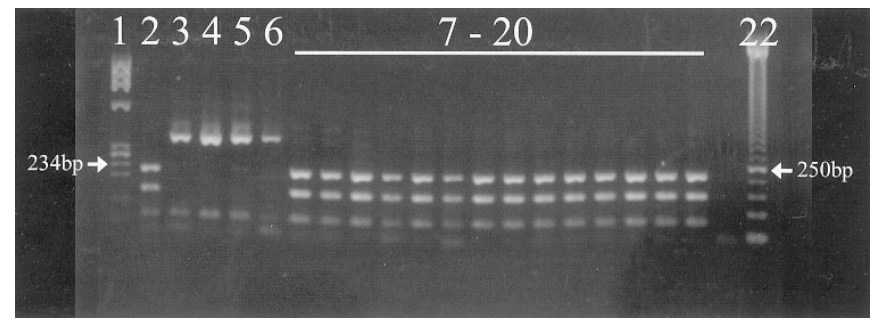

Fig. 5 Agarose gel analysis of specimens digested with MnlI. Samples are as follows: Lane 1, ФX174-HaeIII molecular weight marker; Lane 2, wild type specimen; Lanes 3 and 4, specimens carrying T961G; Lanes 5 and 6, specimens carrying 961delT + C(n); Lane 7, specimen carrying 956-960insC; Lanes 8-20, specimens of unknown sequence; and Lane 22, 50 base pair (bp) ladder molecular weight marker. Lane 21 is a specimen PCR failure that was successfully repeated on another gel. The sequences of the control specimens in Lanes 2-7 were known from the DNA sequencing of suspect ASO results.

Because the restriction enzyme analysis used here is not able to distinguish between the wild type sequence and the 956960insC alteration, it is not known with certainty that no additional occurrences of the 956-960insC sequence exist in this specimen population. However, as no additional occurrences of the other two sequence changes were found by restriction analysis, it is unlikely that many more occurrences of the 956960insC sequence alteration would be found. Furthermore, because the $956-960$ ins $C$ sequence change was not the original sequence of interest, sequencing of the entire specimen population to identify additional occurrences of this sequence has not been pursued.

Specimens found by sequencing to carry the $961 \mathrm{delT}+$ $\mathrm{C}(\mathrm{n})$, T961G, and 956-960insC sequence changes were as- sessed for mitochondrial haplogroup in an effort to provide insight into the mitochondrial backgrounds on which these sequence changes could be found. ${ }^{17-24}$ All six specimens carrying the T961G sequence change demonstrate loss of the AluI site at position 7025 of the mitochondrial DNA, characteristic of Haplogroup H. One of the five specimens carrying the 956960insC sequence change was found to have an NlaIII site gain at position 4216 and a BamHI site gain at position 13366, characteristic of Haplogroup T. Another of the specimens carrying the 956-960insC mutation displayed gain of a HaeIII site at position 663, characteristic of Haplogroup A. This specimen also demonstrated gain of an AvaII site at position 8249, as seen in Haplogroups W and I but lacked other site gains and losses required for placement in those haplogroups. The remaining three specimens carrying the 956-960insC mutation remain uncategorized and are of unknown haplogroup. Of the seven specimens carrying the 961delT $+\mathrm{C}(\mathrm{n})$ mutation, one specimen demonstrated gain of a $\mathrm{HpaI}$ site at position 3592, placing it in Haplogroup L; one specimen demonstrated gain of a HaeIII site at position 663, placing it in Haplogroup A; one specimen demonstrated the 9 base pair deletion between positions 8269 and 8295, characteristic of Haplogroup B; three specimens demonstrated gain of a DdeI site at position 10394 with gain of an $A l u$ I site at position 10397, placing them in Haplogroup M; and one specimen remains uncategorized after haplogroup analyses and is of unknown haplogroup (data not shown).

To provide a general context for the study population, data about the gender distribution and ethnicity of births and the 
rate of multiple births in the state of Texas was obtained from the Texas Department of Health Bureau of Vital Statistics web site (http://www.tdh.state.tx.us/bvs). Although the specimens for this study were collected over a period of less than 1 year, data from the three most recently available years—1997, 1998, and 1999 - were collected and averaged to provide a reasonable estimation of the demographic characteristics of this study's subject population (Table 1). At the time of preparation of this manuscript, the year 2000 data were considered preliminary by the Texas Department of Health. These data are included in Table 1 for comparison (in italics), but were not used in the calculations. Year 2001 data were not yet available.

The Texas Bureau of Vital Statistics web site (http://www.tdh.state.tx.us/bvs) also provides data on multiple birth rates in Texas. Twin births generally account for $<2.6 \%$ of births in Texas annually. Triplet and higher order births account for approximately $0.1 \%$ of births in Texas annually. Therefore, multiple births should make up $<2.7 \%$ of this study population. Statistically, the specimen population in this study should contain $<15$ sets of twins and $<1$ set of higher order births. Inasmuch as the mutations analyzed here are mitochondrial, specimens from multiple births would be expected to share genotypes at the loci examined. Because of the limited quantity of each individual specimen, identity testing to assess this subject population for twin or higher order births was not performed.

These data suggest the carrier frequency for the A1555G mutation may be as high as $0.09 \%$ of the overall Texas population and the carrier frequency for the 961delT $+C(n)$ mutation may be $0.6 \%$. These data also provide a preliminary indication that the 961delT $+\mathrm{C}(\mathrm{n})$ mutation occurs in more than one mitochondrial haplogroup and in more than one ethnic group. Based on these data, approximately $2,300-2,400$ children carrying one of these mutations could be born each year in Texas.

\section{DISCUSSION}

A large unselected Texas population was tested for the presence of the A1555G and 961delT $+\mathrm{C}(\mathrm{n})$ mutations. The A1555G mutation was found in 1 specimen out of 1,161 . The 961delT $+\mathrm{C}(\mathrm{n})$ mutation was found in 7 specimens. Two previously unreported mutations, T961G and 956-960insC, were found in 6 and 5 specimens, respectively.

The number of specimens carrying the 961delT $+C(n)$ mutation was unexpected. These data suggest a carrier frequency for this mutation of $\sim 1$ in 166 in the Texas population. Furthermore, the observation of two additional nucleotide changes at or around position 961, T961G and 956-960insC, implies that this may be a far more variable region of the mitochondrial genome than previously suspected. The data suggest that in some populations, $\sim 1$ in 65 persons may carry some variability in sequence in this region of the mitochondrial genome.

The results of haplogroup analyses show that the seven occurrences of the 961delT $+C(n)$ sequence change observed in this study occur in at least five different haplogroups including the African L haplogroup, the Asian M haplogroup, the Native American/Asian A and B haplogroups, and at least one other mitochondrial haplogroup. These data also show that the 956960insC sequence change occurs in the Caucasian T haplogroup, the Native American/Asian A haplogroup, and at least one other mitochondrial haplogroup. These data suggest that these two mutations may have occurred more than once in human history and multiple ethnic groups may be at risk for carrying these mutations. The observation that the T961G sequence change occurs only in the Caucasian $\mathrm{H}$ haplogroup in this study suggests that this mutation may be more common in Caucasian populations than in other groups.

It is not known whether any of these sequence changes occurred in multiple births. However, the specimens for this study were collected in two batches, with less than a year elapsed time between isolation of the batches. As such, the rate of multiple births in the study population is expected to be low and should reflect the annual rate of multiple births in Texas, which is $<2.7 \%$. Only a single occurrence of the A1555G mutation was found, suggesting that a twin of that specimen was not included in the study population. Furthermore, haplogroup analysis of specimens carrying the $961 \mathrm{delT}+\mathrm{C}(\mathrm{n}) \mathrm{mu}-$ tation, a primary sequence of interest in this study, suggests that the seven specimens carrying this mutation are distributed among at least five different haplogroups. As a result, it is unlikely that the higher than expected rate of occurrence of the

Table 1

Texas births, 1997-2000

\begin{tabular}{|c|c|c|c|c|c|c|}
\hline Year & $\begin{array}{r}\text { Total } \\
\text { births }\end{array}$ & $\begin{array}{l}\text { Males/ } \\
\text { females }\end{array}$ & Whites & Blacks & Hispanics & Other \\
\hline 1997 & 333,829 & 1.05 & 137,796 & 39,406 & 146,147 & 10,480 \\
\hline 1998 & 342,199 & 1.04 & 140,325 & 40,123 & 151,116 & 10,635 \\
\hline 1999 & 349,157 & 1.05 & 140,374 & 40,005 & 157,329 & 11,449 \\
\hline 2000 & 363,325 & 1.04 & $142,553(39 \%)$ & $41,180(11 \%)$ & $166,440(46 \%)$ & $13,152(4 \%)$ \\
\hline 3 -year averages & 341,728 & 1.05 & $139,498(41 \%)$ & $39,845(12 \%)$ & $151,531(44 \%)$ & $10,855(3 \%)$ \\
\hline
\end{tabular}

Column averages, excluding year 2000 data, are provided in the last row. Data were obtained January 2-3, 2002, from the Texas Department of Health Bureau of Vital Statistics web site (http://www.tdh.state.tx.us/bvs). Preliminary data for the year 2000 in italics. 
961delT $+C(n)$ mutation can be explained entirely by clustering of multiple births within this subpopulation of specimens.

To clarify why the 961delT $+\mathrm{C}(\mathrm{n})$ mutation was not found in 799 controls in a previous study by others, ${ }^{4}$ but was found at such a high frequency in this population, that report and its referenced articles $4,18,19,25-28$ were revisited. In those studies, 699 of the controls cited were evaluated by restriction mapping methods of large PCR fragments for haplogroup analysis. The restriction enzymes used in those studies would not have been directly affected by the sequence changes at and around position 961 described here. As a result, no loss or gain of a restriction site would have occurred and no obvious changes in the restriction patterns would have been observed by the agarose gel detection methods used. It is to be expected that the T961G alteration would not have been detected by these methods. Furthermore, the 956-960insC sequence change, a single nucleotide insertion, would likely have been too small a size difference to be seen by the agarose gel methods that were used. Finally, the 961delT $+\mathrm{C}(\mathrm{n})$, which represents a change of only $8-15$ nucleotides in length, may also have been too small to be detected by the limited sensitivity of the agarose gel methods used in those studies. The haplogroup analyses conducted in this study support this explanation. The specimens carrying these different mutations appeared identical on agarose gelbased haplogroup analysis, with the exception of haplogroupspecific restriction site gains and losses. An alternative explanation is that the ethnic variability between the specimens in those studies ${ }^{4,18,19,25-28}$ and the specimen population described here accounts for the observations. However, given the diversity of the subject population used in this study, the variety of haplogroups on which the observed mutations occur, and the frequency with which changes appear to occur at and around nucleotide 961, this explanation seems less likely.

As demonstrated by these data, ASO, while effective for detection of the A1555G mutation, is not an effective screening methodology for detection of the 961delT $+\mathrm{C}(\mathrm{n})$ mutation. Technical difficulty in detection the 961delT $+\mathrm{C}(\mathrm{n})$ mutation by ASO was also noted by Bacino et al. ${ }^{3}$ It is possible that the technical problem with ASO detection of this mutation may be related to the sequence of the mutant oligonucleotide probe. The oligonucleotides used in this study and the Bacino et al. study ${ }^{3}$ included additional sequence $5^{\prime}$ and $3^{\prime}$ of the CCCCCTCCCC segment. Any of the sequence changes observed in this study would result in mismatches between the probe oligonucleotide and the target DNA sequence, likely resulting in a failure to bind under stringent conditions. No attempt was made in this study to optimize the probe oligonucleotide design or assay conditions to develop a more successful ASO assay. Instead, the restriction enzyme MnlI was used to screen the subject population for the 961delT $+C(n)$ and T961G sequence alterations. The restriction enzyme MnlI will not, however, detect the 956-960insC alteration. Specimens carrying this sequence change appear as wild type after restriction digestion, requiring DNA sequencing for detection.

The $\mathrm{A} 1555 \mathrm{G}$ mutation is known to occur in a variety of ethnic groups, on a variety of mitochondrial backgrounds, and has been documented in populations around the globe. This is the first study to screen a large, unselected, ethnically diverse population for genetic susceptibility to aminoglycoside ototoxicity. Prior studies ${ }^{1-15}$ have suggested that, outside the context of hereditary hearing impairment, the $\mathrm{A} 1555 \mathrm{G}$ mutation is rare. However, if future studies confirm the approximate carrier rate for the $\mathrm{A} 1555 \mathrm{G}$ mutation of $0.09 \%$ found in this study, hundreds of thousands of people in the United States, and many more around the globe, are potentially at risk for aminoglycoside ototoxicity: far more than are at risk for the diseases detected by current newborn screening programs. Precise estimation of any given individual's risk for aminoglycoside exposure is difficult and may be confounded by a variety of geographic, environmental, and immunologic factors; however, given the perception that use of aminoglycoside antibiotics in the United States is reduced compared with other countries, the most profound impact of carrier estimations for the A1555G mutation may be outside the United States, where A1555G carriers may be at greater lifetime risk for exposure to aminoglycoside antibiotics than are those who live in the United States.

The rapid and efficient ASO methodology for detection of the A1555G mutation combined with the knowledge that the mutation occurs in a wide variety of ethnic groups and the incidence data from this study suggest that some type of screening for this mutation may warrant further discussion. Knowing an individual is at risk for aminoglycoside ototoxicity may provide an opportunity to prevent acquired hearing loss through avoidance of aminoglycoside antibiotics, thereby possibly delaying the onset of hearing loss for many years. ${ }^{8}$ Knowing an individual carries the A1555G mutation would also allow for genetic counseling and avoidance of aminoglycosides in certain relatives. Furthermore, knowing an individual's carrier status may provide an opportunity for anticipatory care including medical, educational, and occupational interventions relevant to hearing loss. ${ }^{29,30}$

Unfortunately, knowledge of carrier status will not likely prevent hearing loss in all mutation carriers because of the mutation's clear role in cases of hearing loss that lack exposure to aminoglycoside antibiotics (50\% penetrance by age 30, $88 \%$ penetrance by age 65). ${ }^{8}$ In addition to hearing loss, there has been a single report of maternally inherited cardiomyopathy in a family carrying the A1555G mutation in a heteroplasmic state. ${ }^{31}$ These observations raise questions about which are the best populations for screening and how informed consent should be obtained prior to screening. Furthermore, because a single occurrence of the A1555G mutation was found in this population, additional large-population studies are needed to refine its occurrence in the US population.

Because of the anonymity of this study's subject population, phenotypic information and outcome data including medical records, hearing status, and history of exposure to aminoglycoside antibiotics are unavailable for the mutation carriers detected. It is also impossible in a study such as this to follow subjects over time. Furthermore, it is not known whether carriers of the previously unreported sequence changes might also 
be at risk for aminoglycoside-induced hearing loss or some other mitochondrial disorder or whether these are simply benign polymorphisms. However, the higher than expected rate of occurrence of changes at and around position 961 of the mitochondrial $12 \mathrm{~S}$ rRNA leads one to speculate that perhaps the 961delT $+\mathrm{C}(\mathrm{n})$ mutation is not associated with aminoglycoside ototoxicity and was coincidentally found in the reported cases. ${ }^{3,4}$ Alternatively, perhaps this mutation carries reduced penetrance for conferring susceptibility to aminoglycoside-induced ototoxicity and additional genetic and/or environmental factors are required for hearing loss to occur. Further studies are clearly needed to address whether and how often this mutation predisposes carriers to aminoglycoside ototoxicity. If future studies of the 961delT $+\mathrm{C}(\mathrm{n})$ sequence change confirm its role in aminoglycoside ototoxicity, the data reported here suggest that it may be a far more common cause for genetic susceptibility to aminoglycoside-induced hearing loss than previously suspected, perhaps as much as seven times more common than the $\mathrm{A} 1555 \mathrm{G}$ mutation. However, if the changes at or around position 961 are found to be unrelated to risk for aminoglycoside ototoxicity, these mutations should be excluded from screening or testing protocols for individual risk of hearing loss from aminoglycoside antibiotics.

Additional characterization of the physiologic consequences of both the A1555G and 961delT $+\mathrm{C}(\mathrm{n})$ mutations is needed to understand the full effects of these mutations with and without aminoglycoside exposure and to identify the variability of additional medical risks that may need to be addressed. Future studies will need to be designed to identify large numbers of individuals with a known history of aminoglycoside exposure for genotype/phenotype and haplogroup analyses. Data on the prevalence of aminoglycoside use in the population are also needed. Data from such studies will provide information about the clinical utility of testing for genetic susceptibility to aminoglycoside ototoxicity and will support discussions of screening for genetic susceptibility to aminoglycoside ototoxicity by identifying high-risk populations. Data from these types of studies will also help establish guidelines for pretest genetic counseling and informed consent of subjects.

\section{Acknowledgments}

This work was supported by the Allbritton Fund and the Brown Foundation. The authors gratefully acknowledge the assistance of the following individuals: from the Texas Department of Health, Lynette Borgfeld and Lori Durham provided assistance with the collection, anonymization, and shipping of dried blood spots, and Sharilyn K. Stanley, MD, provided critical review of the manuscript; from Baylor College of Medicine, Ellen M. Friedman, MD, Bobby R. Alford, MD, and Aletta Moore provided critical review of the manuscript.

\section{References}

1. Prezant TR, Agapian JV, Bohlman MC, Bu X, Oztas S, Qiu WQ, Arnos KS, Cortopassi GA, Jaber L, Rotter JI, et al. Mitochondrial ribosomal RNA mutation associ- ated with both antibiotic-induced and nonsyndromic deafness. Nat Genet 1993;4: 289-294.

2. Hutchin T, Haworth I, Higashi K, Fischel-Ghodsian N, Stoneking M, Saha N, Arnos C, Cortopassi G. A molecular basis for human hypersensitivity to aminoglycoside antibiotics. Nucleic Acids Res 1993;21:4174-4179.

3. Bacino C, Prezant TR, Bu X, Fournier P, Fischel-Ghodsian N. Susceptibility mutations in the mitochondrial small ribosomal RNA gene in aminoglycoside induced deafness. Pharmacogenetics 1995;5:165-172.

4. Casano RAMS, Johnson DF, Bykhovskaya Y, Torricelli F, Bigozzi M, Fischel-Ghodsian N. Inherited susceptibility to aminoglycoside ototoxicity: genetic heterogeneity and clinical implications. Am J Otolaryngol 1999;20:151-156.

5. Fischel-Ghodsian N, Prezant TR, Bu X, Oztas S. Mitochondrial ribosomal RNA gene mutation in a patient with sporadic aminoglycoside ototoxicity. Am J Otolaryngol 1993;14:399-403.

6. Gardner JC, Goliath R, Viljoen D, Sellars S, Cortopassi G, Hutchin T, Greenberg J, Beighton P. Familial streptomycin ototoxicity in a South African family: a mitochondrial disorder. J Med Genet 1997;34:904-906.

7. Pandya A, Xia X, Radnaabazar J, Batsuuri J, Dangaansuren B, Fischel-Ghodsian N, Nance WE. Mutation in the mitochondrial 12S rRNA gene in two families from Mongolia with matrilineal aminoglycoside ototoxicity. J Med Genet 1997;34:169172.

8. Estivill X, Govea N, Barcelo E, Badenas C, Romero E, Moral L, Scozzri R, D’Urbano L, Zeviani M, Torroni A. Familial progressive sensorineural deafness is mainly due to the mtDNA A1555G mutation and is enhanced by treatment with aminoglycosides. Am J Hum Genet 1998;62:27-35.

9. Shohat M, Fischel-Ghodsian N, Legum C, Halpern GJ. Aminoglycoside-induced deafness associated with the mitochondrial DNA mutation A1555G. Am J Otolaryngol 1999;20:64-67.

10. Matthijs G, Claes S, Longo-Mbenza B, Cassiman J-J. Non-syndromic deafness associated with a mutation and a polymorphism in the mitochondrial $12 \mathrm{~S}$ ribosomal RNA gene in a large Zairean pedigree. Eur J Hum Genet 1996;4:46-51.

11. Casano RA, Bykhovskaya Y, Johnson DF, Hamon M, Torricelli F, Bigozzi M, Fischel-Ghodsian N. Hearing loss due to the mitochondrial A1555G mutation in Italian families. Am J Med Genet 1998;79:388-391.

12. Usami S, Abe S, Kasai M, Shinkawa H, Moeller B, Kenyon JB, Kimberling WJ. Genetic and clinical features of sensorineural hearing loss associated with the 1555 mitochondrial mutation. Laryngoscope 1997;107:483-490.

13. Usami S, Abe S, Akita J, Namba A, Shinkawa H, Ishii M, Iwasaki S, Hoshino T, Ito J, Doi K, Kubo T, Nakagawa T, Komiyama S, Tono T, Komune S. Prevalence of mitochondrial gene mutations among hearing impaired patients. J Med Genet 2000; 37:38-40.

14. Fischel-Ghodsian N, Prezant TR, Chaltraw WE, Wendt KA, Nelson RA, Arnos KS, Falk RE. Mitochondrial gene mutation is a significant predisposing factor in aminoglycoside ototoxicity. Am J Otolarynol 1997;18:173-178.

15. Scrimshaw BJ, Faed JM, Tate WP, Yun K. The frequency in New Zealand of a mitochondrial DNA mutation (1555 A to G) associated with aminoglycoside-induced hearing loss. N Z Med J 1999;112:216-217.

16. Torroni A, Cruciani F, Rengo C, Sellitto D, Lopez-Bigas N, Rabionet R, Govea N, Lopez De Munain A, Sarduy M, Romero L, Villamar M, del Castillo I, Moreno F, Estivill X, Scozzari R. The A1555G mutation in the 12S rRNA gene of human mtDNA: recurrent origins and founder events in families affected by sensorineural deafness. Am J Hum Genet 1999;65:1349-1358.

17. MITOMAP. A human mitochondrial genome database. Atlanta, GA: Center for Molecular Medicine, Emory University, 2001. http://www.gen.emory.edu/mitomap.html.

18. Ballinger SW, Schurr TG, Torroni A, Gan YY, Hodge JA, Hassan K, Chen KH, Wallace DC. Southeast Asian mitochondrial DNA analysis reveals genetic continuity of ancient Mongoloid migrations. Genetics 1992;130:139-152.

19. Torroni A, Schurr TG, Yang CC, Szathmary EJ, Williams RC, Schanfield MS, Troup GA, Knowler WC, Lawrence DN, Weiss KM, et al. Native American mitochondrial DNA analysis indicates that the Amerind and the Nadene populations were founded by two independent migrations. Genetics 1992;130:153-162.

20. Torroni A, Lott MT, Cabell MF, Chen YS, Lavergne L, Wallace DC. mtDNA and the origin of Caucasians: identification of ancient Caucasian-specific haplogroups, one of which is prone to a recurrent somatic duplication in the D-loop region. Am J Hum Genet 1994;55:760-776.

21. Torroni A, Huoponen K, Francalacci P, Petrozzi M, Morelli L, Scozzari R, Obinu D, Savontaus ML, Wallace DC. Classification of European mtDNAs from an analysis of three European populations. Genetics 1996;144:1835-1850.

22. Macaulay V, Richards M, Hickey E, Vega E, Cruciani F, Guida V, Scozzari R, Bonne-Tamir B, Sykes B, Torroni A. The emerging tree of West Eurasian mtDNAs: a synthesis of control-regions sequences and RFLPs. Am J Hum Genet 1999;64:232-249. 
23. Morelli L, Grosso MG, Vona G, Varesi L, Torroni A, Francalacci P. Frequency distribution of mitochondrial DNA haplogroups in Corsica and Sardinia. Hum Biol 2000;72:585-595.

24. Ruiz-Pesini E, Lapena AC, Diez-Sanchez C, Perez-Martos A, Montoya J, Alvarez E, Diaz M, Urries A, Montoro L, Lopez-Perez MJ, Enriquez JA. Human mtDNA haplogroups associated with high or reduced spermatozoa motility. Am J Hum Genet 2000;67:682-696.

25. Shoffner JM, Brown MD, Torroni A, Lott MT, Cabell MF, Mirra SS, Beal MF, Yang CC, Gearing M, Salvo R, et al. Mitochondrial DNA variants observed in Alzheimer disease and Parkinson disease patients. Genomics 1993;17:171-184.

26. Brown MD, Voljavec AS, Lott MT, Torroni A, Yang C-C, Wallace DC. Mitochondrial DNA complex I and III mutations associated with Leber's hereditary optic neuropathy. Genetics 1992;130:163-173.
27. Cann RL, Stoneking M, Wilson AC. Mitochondrial DNA and human evolution. Nature 1987;325:31-36.

28. Stoneking M, Jorde LB, Bhatia K, Wilson AC. Geographic variation in human mitochondrial DNA from Papua New Guinea. Genetics 1990;124:717-733.

29. Therrell B. US newborn screening policy dilemmas for the twenty-first century. Mol Genet Metab 2001;74:64-74.

30. Tono T, Ushisako Y, Kiyomizu K, Usami S, Abe S, Shinkawa H, Komune S. Cochlear implantation in a patient with profound hearing loss with the A1555G mitochondrial mutation. Am J Otol 1998;19:754-757.

31. Santorelli FM, Tanji K, Manta P, Casali C, Krishna S, Hays AP, Mancini DM, DiMauro S, Hirano M. Maternally inherited cardiomyopathy: an atypical presentation of the mtDNA $12 \mathrm{~S}$ rRNA gene A1555G mutation. Am J Hum Genet 1999;64:295-300. 